KAIST-TH 99/02, SNUTP-99-9,hep-ph/9902291

\title{
Cosmological Gravitino Production in Gauge Mediated Supersymmetry Breaking Models
}

\author{
Kiwoon Choi, Kyuwan Hwang, Hang Bae Kim \\ Department of Physics, Korea Advanced Institute of Science and Technology \\ Taejon 305-701, Korea \\ Taekoon Lee \\ Center for Theoretical Physics, Seoul National University \\ Seoul 151-742, Korea
}

\begin{abstract}
We study the cosmological gravitino production in gauge mediated supersymmetry breaking models, while properly taking into account the existence of the messenger mass scale. It is found that for sizable parameter range of the model the messenger sector contribution leads to more stringent upper bound on the reheat temperature obtained from the condition that the universe should not be overclosed by relic gravitinos. However it turns out that in the limit of relatively low messenger scale and large gravitino mass, the relic gravitino mass density can be smaller than the critical density independently of the reheat temperature, suggesting the possibility in this limit to have a high reheat temperature without the dilution of gravitinos at late time.
\end{abstract}

Typeset using REVTEX 


\section{INTRODUCTION}

Supersymmetry (SUSY) is an elegant solution to the mass hierarchy problem of the standard model [1]. But it must be spontaneously broken because we do not see degenerate pairs of particles and their superpartners. Within the minimal supersymmetric standard model (MSSM), it seems unavoidable to introduce explicit supersymmetry breaking through soft breaking terms. Such soft breaking terms might be a consequence of spontaneous supersymmetry breaking which is transmitted to the MSSM sector by certain mediating interactions. In this regards, there are two classes of models which are distinguished by the nature of mediating interactions: gravity-mediated models [囯] and gauge-mediated models [2].

Cosmological consequences of supersymmetry breaking models have been also discussed to some extent. One of the major issues has been the relic abundance of the lightest supersymmetric particle (LSP). In gauge-mediated models, one interesting feature is that the gravitino is very light and thus becomes the LSP. For a light gravitino, its interaction is dominated by the longitudinal goldstino component whose interaction strength is proportional to $1 / m_{3 / 2}$ and thus gets stronger as the gravitino mass $m_{3 / 2}$ becomes smaller [3]. Then the considerable amount of light gravitinos can be produced from the decay or collisions of the MSSM particles, even in the case that the gravitino number density was negligible just after the reheating phase of inflation. Not to overclose the universe, this imposes a strong constraint on the reheat temperature depending upon the gravitino mass, unless the gravitinos produced in this way were diluted by a late time entropy production [4.5].

In the previous studies [4,5], only the gravitino productions by the MSSM particles were considered under the assumption that the local goldstino couplings to the MSSM fields are valid up to energy scales around the reheat temperature $T_{R}$. However it was observed by one of the authors (T.L.) that gravitinos decouple from the MSSM fields at temperatures above the messinger scale at which the gravitino interaction to the MSSM particles is induced radiatively [6]. In this paper, we reexamine the cosmological gravitino production while properly taking into account the existence of the new mass scale in gaugemediated models, i.e. the messenger scale $M_{X}$, which is deeply involved in the problem. We first note that the goldstino production due to the MSSM particles at high temperature $T>M_{X}$ is suppressed by $M_{X}^{2} / T^{2}$ when compared to the naive extrapolation of the low temperature results at $T<M_{X}$. However, in this high temperature range the messenger sector contribution is much larger than that of the MSSM particles because the messenger particles have stronger couplings to the goldstino. As a result, for much of the parameter range of $\left(M_{X}, m_{3 / 2}\right)$, the messenger sector contribution leads to more stringent upper bound on the reheat temperature which is obtained from the condition that the universe should not be overclosed by relic gravitinos. The results are depicted in Fig. 2 for several values of the messenger and MSSM gaugino masses.

An interesting feature of the messenger sector contribution is that the relic gravitino number density to the entropy number density ratio $\left(Y_{3 / 2} \equiv n_{3 / 2}(T) / s(T)\right)$ behaves such as $d Y_{3 / 2} \propto d T /\left(m_{3 / 2}^{2} T^{n}\right)(n \geq 2)$ for $M_{X}<T<T_{R}$, indicating that the present value of $Y_{3 / 2}$ is mostly induced at $T \sim M_{X}$, not at the highest available temperature $T \sim T_{R}$. As a result, in gauge-mediated models with $M_{X} \lesssim T_{R}$, the gravitino abundance is determined by the values of $m_{3 / 2}$ and $M_{X}$, and is independent of $T_{R}$. This means that in the limit 
of relatively low messenger scale and large gravitino mass, the relic gravitino mass density can be smaller than the critical density independently of $T_{R}$. Of course, this would be true only when other more model-dependent contributions, e.g. those from the supersymmetry breaking sector, are negligible, which would be the case if the typical mass scale of the supersymmetry breaking sector is bigger than $T_{R}$. The results of Eq.(19) and Fig. 2 show that for the messenger mass $M_{X}=10^{5} \sim 10^{6} \mathrm{GeV}$ and the gravitino mass $m_{3 / 2} \gtrsim 100$ $\mathrm{MeV}$, we can have $T_{R}$ much higher than the previously obtained bound without assuming the dilution of gravitinos at late time.

\section{GOLDSTINO COUPLINGS IN GAUGE-MEDIATED MODELS}

Gauge mediated supersymmetry breaking (GMSB) models consist of the observable (MSSM) sector, the messenger sector, and also the supersymmetry breaking sector [2]. Supersymmetry breaking sector breaks supersymmetry dynamically at certain high energy scales and delivers supersymmetry breaking effects to the messenger sector through messenger gauge interactions [7] or other interactions [8,9]. Supersymmetry breaking in the messenger sector is finally transmitted to the MSSM sector via the standard model gauge interactions and generates the soft supersymmetry breaking masses of order $10^{2} \sim 10^{3} \mathrm{GeV}$. After integrating out the supersymmetry breaking sector, the effective superpotential is given by

$$
W_{\mathrm{eff}}=\lambda X \Phi \Phi^{c}+\Delta W\left(X, Y_{i}\right)
$$

where $X$ and $Y_{i}$ are the standard model singlets and the nonperturbative effects of the supersymmetry breaking sector are encoded in $\Delta W$. Here we consider the simplest messenger sector, consisting of single flavor of chiral superfield $\Phi+\Phi^{c}$ transforming as $5+\overline{5}$ of $S U(5)$. By minimizing the effective potential from $W_{\text {eff }}$, we can determine the vacuum expectation values (VEVs) $\langle X\rangle,\left\langle Y_{i}\right\rangle$ and also $\left\langle F_{X}^{*}\right\rangle=\left\langle\partial W_{\text {eff }} / \partial X\right\rangle,\left\langle F_{i}^{*}\right\rangle=\left\langle\partial W_{\text {eff }} / \partial Y_{i}\right\rangle$ which measure the size of supersymmetry breaking. The $\mathrm{VEV}\langle X\rangle$ gives a supersymmetric mass $\lambda\langle X\rangle \equiv M_{X}$ to the messenger $\Phi+\Phi^{c}$, while $\left\langle F_{X}^{*}\right\rangle$ leads to the supersymmetry breaking mass splitting in the scalar masses of $\Phi+\Phi^{c}$. Supersymmetry breaking in the messenger sector is transmitted to the MSSM particles via the standard model gauge interactions at one-loop or two-loop levels, yielding the supersymmetry breaking mass splitting in MSSM particles:

$$
m_{\lambda} \sim(\alpha / 4 \pi) F_{X} /\langle X\rangle, \quad m_{\tilde{f}}^{2} \sim(\alpha / 4 \pi)^{2}\left(F_{X} /\langle X\rangle\right)^{2},
$$

where $m_{\lambda}$ and $m_{\tilde{f}}$ denote the soft gaugino mass and scalar mass, respectively, and $\alpha$ is the MSSM fine structure constant.

The spontaneous breakdown of supersymmetry leads to a massless spin- $1 / 2$ fermions, the goldstino. The goldstino field is a linear combination of the fermion components of $X$ and $Y_{i}$ weighted by $F_{X}$ and $F_{i}$, i.e.,

$$
\chi=\frac{\sum_{I} F_{I}^{*} \psi_{I}}{F}
$$

where $F=\sqrt{\left|F_{X}\right|^{2}+\sum_{i}\left|F_{i}\right|^{2}}$. When global supersymmetry is promoted to local supersymmetry (supergravity), the goldstino is absorbed into the gravitino as the longitudinal components and the gravitino acquires a mass 


$$
m_{3 / 2}=\frac{F}{\sqrt{3} M_{P}}
$$

with the vanishing cosmological constant assumed.

At energy scales below $\sqrt{F} \sim \sqrt{m_{3 / 2} M_{P}}$ but above the messenger mass scale $M_{X}=$ $\lambda\langle X\rangle$, the goldstino $\chi$ directly couples to the messenger fields, which can be read off from the superpotential (11) using the relation $\psi_{I}=\left(F_{I} / F\right) \chi+\cdots$,

$$
\begin{aligned}
\mathcal{L}_{\text {messenger }} & =\lambda \frac{F_{X}}{F}\left(\phi \chi \psi^{c}+\phi^{c} \chi \psi\right)+\cdots \\
& \sim \frac{4 \pi}{\alpha} \frac{m_{\lambda} M_{X}}{\sqrt{3} m_{3 / 2} M_{P}}\left(\phi \chi \psi^{c}+\phi^{c} \chi \psi\right)+\cdots,
\end{aligned}
$$

where $\phi+\phi^{c}$ and $\psi+\psi^{c}$ denote the scalar and fermion components of the messenger superfields $\Phi+\Phi^{c}$. At these energy scales, there is no direct local couplings of the goldstino to the MSSM particles at lagrangian level. However at lower energy scales below $M_{X}$, one can show [6] that integrating out the messenger particles through one and two loop diagrams leads to the following effective lagrangian [3, 10, 11]:

$$
\mathcal{L}_{M S S M}=\frac{m_{\tilde{f}}^{2}-m_{f}^{2}}{\sqrt{3} m_{3 / 2} M_{P}} \bar{f} \chi \tilde{f}^{*}+\frac{m_{\lambda}}{4 \sqrt{6} m_{3 / 2} M_{P}} \bar{\chi} \sigma_{\mu \nu} \lambda F^{\mu \nu}-\frac{g m_{\lambda}}{\sqrt{6} m_{3 / 2} M_{P}} \tilde{f} \tilde{f}^{*} \chi \lambda+\text { h.c. }
$$

where $(\tilde{f}, f)$ and $\left(\lambda, F_{\mu \nu}\right)$ denote the MSSM matter and gauge multiplets, respectively. We stress that the above local goldstino couplings to the MSSM fields are valid only at energy scales below $M_{X}$. When computing the amplitudes with an external energy $E \geq M_{X}$ (but still below $\sqrt{F}$ ), one has to use (5), not (6), even when the external particles involve only the goldstino and MSSM particles. Note that the effective lagrangian (6) summarizes the leading order results of the low energy amplitudes expanded in powers of $E / M_{X}$.

For the soft masses (2) presumed to be of order $10^{2} \sim 10^{3} \mathrm{GeV}$, goldstino couplings are described essentially by the two unknown model-dependent parameters, $m_{3 / 2}$ and $M_{X}$. In the next section, we will compute the relic gravitino mass density as a function of $m_{3 / 2}$, $M_{X}$, and also the reheat temperature $T_{R}$. The size of $m_{3 / 2}$ in GMSB models has a wide range of model-dependence, which can range from $\mathcal{O}(1)$ eV even to $\mathcal{O}(10) \mathrm{GeV}$. Moreover, depending on how the messenger scale is generated and also on how supersymmetry breaking is transmitted from the supersymmetry breaking sector to the messenger sector, it is possible

to have $M_{X}$ and $\sqrt{F_{X}}$ much smaller than $\sqrt{F} \sim \sqrt{m_{3 / 2} M_{P}}$. (Note that $F_{X} / M_{X}$ is fixed to be of order $4 \pi m_{\lambda} / \alpha \sim 5 \times 10^{4} \mathrm{GeV}$.) This is an interesting possibility in connection with allowing a large reheat temperature, which will be discussed in the next section.

\section{COSMOLOGICAL GRAVITINO PRODUCTION}

At first, let us estimate the temperature range for which the gravitinos are thermalized. For the gravitinos to be thermalized, their reaction rate is required to be larger than the expansion rate of the universe. The expansion rate of the universe is measured by the Hubble parameter $H(T)=\left(\pi^{2} g_{*} / 90\right)^{1 / 2} T^{2} / M_{P}$ for the radiation dominated universe. For 
the temperature below the supersymmetry breaking scale $\sqrt{F} \sim \sqrt{m_{3 / 2} M_{P}}$, the reaction rate of the gravitinos is roughly given by

$$
\Gamma(T) \sim \sum_{I}\left\langle\sigma_{I}\right\rangle n_{I}(T)
$$

where $\left\langle\sigma_{I}\right\rangle$ is the thermal averaged cross section producing the gravitino and $n_{I}(T)$ is the number density. For $T \lesssim M_{X}$, the number density of the messenger particles is Boltzmannsuppressed and then we have to consider only the contributions from the MSSM particles, while for $T \gtrsim M_{X}$, the contributions from the messenger particles are dominant. The resulting reaction rate is given by

$$
\Gamma(T) \sim \begin{cases}\xi_{2} \frac{m_{\lambda}^{2}}{m_{3 / 2}^{2} M_{P}^{2}} T^{3} & \text { for } T \lesssim M_{X}, \\ \xi_{4}\left(\frac{4 \pi}{\alpha}\right)^{2} \frac{m_{\lambda}^{2} M_{X}^{2}}{m_{3 / 2}^{2} M_{P}^{2}} T & \text { for } T \gtrsim M_{X},\end{cases}
$$

where $\xi_{2}$ and $\xi_{4}$ are constants of order unity. (For $\xi_{2}, \xi_{4}$ and the cross sections, see the discussions below.) Then from $\Gamma(T)>H(T)$, we obtain the following temperature range

$$
\begin{aligned}
T \gtrsim \frac{40}{\xi_{2}}\left(\frac{m_{3 / 2}}{m_{\lambda}}\right)^{2} M_{P} & \text { for } T \lesssim M_{X}, \\
T \lesssim \frac{\xi_{4}}{40}\left(\frac{4 \pi}{\alpha}\right)^{2}\left(\frac{m_{\lambda}}{m_{3 / 2}}\right)^{2} \frac{M_{X}^{2}}{M_{P}} & \text { for } T \gtrsim M_{X},
\end{aligned}
$$

for which the gravitinos are thermalized. Once the gravitinos were thermalized, the gravitino mass density to the critical density ratio at present is given by [12]

$$
\Omega_{3 / 2} h^{2} \sim\left(\frac{m_{3 / 2}}{\mathrm{keV}}\right)
$$

and so $m_{3 / 2}$ should be less than about $1 \mathrm{keV}$ not to overclose the universe now. (Here $h=H_{0} /(100 \mathrm{~km} / \mathrm{sec} / \mathrm{Mpc})$ for the present Hubble expansion rate $\left.H_{0}.\right)$

The simplest way to avoid the result (10) is to assume that gravitinos had never reached to thermal equilibrium after the inflation, which would allow $m_{3 / 2} \gtrsim 1 \mathrm{keV}$. Even in this case, significant amount of gravitinos can be produced by the decays or collisions that occur in the thermal bath of the early universe subsequent to inflation [13], which usually leads to an upper bound on the inflation reheat temperature. We can calculate the number density of such gravitinos using the Boltzmann equation

$$
\dot{n}_{3 / 2}+3 H n_{3 / 2}=\sum_{I}\left\langle\Gamma_{(I \rightarrow \chi+\cdots)}\right\rangle n_{I}+\sum_{I, J}\left\langle\sigma_{(I+J \rightarrow \chi+\cdots)} v\right\rangle n_{I} n_{J}+\cdots
$$

Introducing $Y_{3 / 2}(T) \equiv n_{3 / 2}(T) / s(T)$ where $s(T)=\left(2 \pi^{2} / 45\right) g_{* s} T^{3}$ is the entropy density, and changing the variable from the time to the temperature, we can integrate the above Boltzmann equation to obtain

$$
Y_{3 / 2} \equiv\left(\frac{n_{3 / 2}}{s}\right)_{0}=\int_{T_{0}}^{T_{R}} d T \frac{\sum_{I}\left\langle\Gamma_{(I \rightarrow \chi+\cdots)}\right\rangle n_{I}+\sum_{I, J}\left\langle\sigma_{(I+J \rightarrow \chi+\cdots)} v\right\rangle n_{I} n_{J}+\cdots}{s(T) H(T) T} .
$$


Here $T_{R}$ is the reheat temperature after the inflation and the RD era formula $-d t / d T=$ $1 / H T$ is used since most contributions come during the radiation dominated era. The evaluation of the integral of (12) for given $\Gamma_{(I \rightarrow \chi+\cdots)}$ and $\sigma_{(I+J \rightarrow \chi+\cdots)}$ will be explained in the appendix. The gravitino mass density produced by the decays or collisions in the thermal bath is then given by

$$
\Omega_{3 / 2} h^{2} \sim 2.8 \times 10^{8} Y_{3 / 2}\left(\frac{m_{3 / 2}}{\mathrm{GeV}}\right)
$$

The processes which dominantly produce the gravitinos depend on the temperature of the thermal bath. For $T \lesssim M_{X}$, MSSM particles are the dominant source of light gravitinos. From the effective lagrangian (的), $\Gamma_{(x \rightarrow y+\chi)}$ and $\sigma_{(x+y \rightarrow z+\chi)}$ where $x, y, z$ are the MSSM particles can be calculated. When summed over all possible channels, the results are [4]

$$
\begin{aligned}
\sum_{x, y} \Gamma_{(x \rightarrow y+\chi)} & =\xi_{1} \frac{m_{\lambda}^{5}}{m_{3 / 2}^{2} M_{P}^{2}}, \\
\frac{1}{2} \sum_{x, y, z} \sigma_{(x+y \rightarrow z+\chi)} & =\xi_{2} \frac{m_{\lambda}^{2}}{m_{3 / 2}^{2} M_{P}^{2}},
\end{aligned}
$$

where $\xi_{1}$ and $\xi_{2}$ are of order 1 . We then find for $m_{\lambda} \lesssim T_{R} \lesssim M_{X}$

$$
\Omega_{3 / 2} h^{2} \sim\left(\frac{m_{3 / 2}}{100 \mathrm{keV}}\right)^{-1}\left[0.58 \xi_{1}\left(\frac{m_{\lambda}}{10^{3} \mathrm{GeV}}\right)^{3}+2.4 \xi_{2}\left(\frac{m_{\lambda}}{10^{3} \mathrm{GeV}}\right)^{2}\left(\frac{T_{R}}{10^{5} \mathrm{GeV}}\right)\right]
$$

For $T \gtrsim M_{X}$, the effective lagrangian (6) which gives the cross section (15) is not valid any more. When computed using the correct lagrangian (5), the cross section $\sigma_{(x+y \rightarrow z+\chi)}(s)$ falls like $1 / s$ for $\sqrt{s}>M_{X}$. As a result, the gravitino production due to the MSSM particles at temperature $T>M_{X}$ is suppressed by $M_{X}^{2} / T^{2}$ when compared to the naive extrapolation of the result obtained from the effective lagrangian (6). Now the gravitinos are produced dominantly from the processes involving the external messenger particles which couple to the goldstino more strongly. The relevant decay widths and scattering cross sections are given by

$$
\begin{aligned}
\sum_{X, X^{\prime}} \Gamma_{\left(X \rightarrow X^{\prime}+\chi\right)}= & \xi_{3}\left(\frac{4 \pi}{\alpha}\right)^{4} \frac{m_{\lambda}^{4} M_{X}}{3 m_{3 / 2}^{2} M_{P}^{2}} \\
\sum_{x, X, X^{\prime}} \sigma_{\left(x+X \rightarrow X^{\prime}+\chi\right)}= & \xi_{4}\left(\frac{4 \pi}{\alpha}\right)^{2} \frac{m_{\lambda}^{2} M_{X}^{2}}{3 m_{3 / 2}^{2} M_{P}^{2}} \\
& \times \frac{2\left(2 s^{2}-3 M_{X}^{2} s+M_{X}^{4}\right)+s\left(s-2 M_{X}^{2}\right) \log \left(s^{2} / M_{X}^{4}\right)}{s\left(s-M_{X}^{2}\right)^{2}}, \\
\frac{1}{2} \sum_{x, X, X^{\prime}} \sigma_{\left(X+X^{\prime} \rightarrow x+\chi\right)}= & \xi_{4}\left(\frac{4 \pi}{\alpha}\right)^{2} \frac{m_{\lambda}^{2} M_{X}^{2}}{3 m_{3 / 2}^{2} M_{P}^{2}} \\
& \times \frac{2 \sqrt{s\left(s-4 M_{X}^{2}\right)}+M_{X}^{2} \log \left(\frac{s-2 M_{X}^{2}-\sqrt{s\left(s-4 M_{X}^{2}\right)}}{s-2 M_{X}^{2}+\sqrt{s\left(s-4 M_{X}^{2}\right)}}\right)}{s\left(s-4 M_{X}^{2}\right)}
\end{aligned}
$$

where $X, X^{\prime}$ represent the messenger particles transforming as $5+\overline{5}$ of $S U(5)$ and 


$$
\xi_{3}=\frac{1}{4 \pi}, \quad \xi_{4}=\alpha_{1}+3 \alpha_{2}+8 \alpha_{3}
$$

It turns out that the most important contribution comes from the messenger particles at $T \sim M_{X}$, and thus we need a more careful evaluation of the production cross sections for $s=\mathcal{O}\left(M_{X}^{2}\right)$. Now the relic gravitino abundance from the messenger particles can be obtained using the equations in the appendix. For $T_{R} \gtrsim M_{X}$, we find

$$
\Omega_{3 / 2} h^{2} \sim\left(\frac{m_{3 / 2}}{\mathrm{GeV}}\right)^{-1}\left[42 \xi_{3}\left(\frac{m_{\lambda}}{10^{3} \mathrm{GeV}}\right)^{4}\left(\frac{M_{X}}{10^{5} \mathrm{GeV}}\right)^{-1}+1.8 \xi_{4}\left(\frac{m_{\lambda}}{10^{3} \mathrm{GeV}}\right)^{2}\left(\frac{M_{X}}{10^{5} \mathrm{GeV}}\right)\right] .
$$

In Fig. 1, we plotted $\Omega_{3 / 2} h^{2}$ including both the MSSM contributions and the messenger contributions as a function of the reheat temperature $T_{R}$ for typical values of the gravitino, messenger and MSSM gaugino masses. It shows that $\Omega_{3 / 2}$ for $T_{R} \lesssim 0.1 M_{X}$ is from the MSSM contribution, while $\Omega_{3 / 2}$ for $T_{R} \gtrsim 0.1 M_{X}$ is essentially from the messenger sector contribution. Note that $\Omega_{3 / 2}$ is almost independent of $T_{R}$ for $T_{R} \gtrsim M_{X}$ as summarized in (19). This is because $Y_{3 / 2} \equiv n_{3 / 2}(T) / s(T)$ produced by the messenger particles behaves such as

$$
d Y_{3 / 2} \propto \frac{d T}{m_{3 / 2}^{2} T^{n}} \quad(n \geq 2)
$$

and so the present value of $Y_{3 / 2}$ is mostly induced at $T \sim M_{X}$, not at the highest available temperature $T_{R}$. This feature requires a more careful treatment of the integral (12) whose integrand takes a nontrivial form for $T \sim M_{X}$.

Now we can obtain an upper bound on $T_{R}$ as a function of the gravitino mass by requiring $\Omega_{3 / 2} h^{2}<1$. We present the results in Fig. 2 for several values of the messenger and MSSM gaugino masses. Summarizing the results, for the gravitino mass range

$$
m_{3 / 2} \lesssim 0.1\left(\frac{m_{\lambda}}{10^{3} \mathrm{GeV}}\right)^{2}\left(\frac{M_{X}}{10^{5} \mathrm{GeV}}\right) \mathrm{MeV}
$$

we find the practically same result as the previous one obtained considering just the MSSM contributions [4]. For larger $m_{3 / 2}$, the messenger sector contribution leads to a significantly stronger bound on the reheat temperature:

$$
T_{R} \lesssim 0.1 M_{X}
$$

for the gravitino mass range

$$
0.1\left(\frac{m_{\lambda}}{10^{3} \mathrm{GeV}}\right)^{2}\left(\frac{M_{X}}{10^{5} \mathrm{GeV}}\right) \mathrm{MeV} \lesssim m_{3 / 2} \lesssim 10^{3}\left(\frac{m_{\lambda}}{10^{3} \mathrm{GeV}}\right)^{2}\left(\frac{M_{X}}{10^{5} \mathrm{GeV}}\right) \mathrm{MeV} .
$$

However no constraint is imposed on $T_{R}$ for the gravitino mass range

$$
m_{3 / 2} \gtrsim 2\left(\frac{m_{\lambda}}{10^{3} \mathrm{GeV}}\right)^{2}\left(\frac{M_{X}}{10^{5} \mathrm{GeV}}\right) \mathrm{GeV},
$$

suggesting that in gauge mediated models with a small messenger mass $\left(M_{X}=10^{5} \sim 10^{6}\right.$ $\mathrm{GeV})$ and also a relatively large gravitino mass $\left(m_{3 / 2} \gtrsim 100 \mathrm{MeV}\right)$, it may be possible to 
have a rather high reheat temperature without the late dilution of relic gravitinos. Of course, this is possible only when other more model-dependent contributions, e.g. those from the supersymmetry breaking sector, are small enough, which would be the case if the typical mass scale of the supersymmetry breaking sector is bigger than $T_{R}$.

Acknowledgments: This work is supported in part by KOSEF Grant 981-0201-004-2, KOSEF through CTP of Seoul National University, KRF under the Grant 1998-015-D00071 and the Distinguished Scholar Exchange Program.

\section{APPENDIX}

In this appendix, we present the formulae for the integral of (12) when the decay width $\Gamma(E)$ and the cross section $\sigma(s)$ are given. The decay and scattering terms in the Boltzmann equation can be written as

$$
\begin{aligned}
\langle\Gamma(E)\rangle n= & \Gamma \frac{m T^{2}}{2 \pi^{2}} \int_{m / T}^{\infty} d x \frac{\left(x^{2}-m^{2} / T^{2}\right)^{1 / 2}}{e^{x} \mp 1} \\
\left\langle\sigma_{(1+2 \rightarrow \cdots)} v\right\rangle n_{I} n_{J}= & \frac{T^{2}}{16 \pi^{4}} \int_{\left(m_{1}+m_{2}\right) / T}^{\infty} d x K_{1}(x) \sigma\left(x^{2} T^{2}\right) \\
& \times\left[\left(x^{2} T^{2}-m_{1}^{2}-m_{2}^{2}\right)^{2}-4 m_{1}^{2} m_{2}^{2}\right],
\end{aligned}
$$

where $\Gamma$ is the decay width in the rest frame and $K_{1}$ is the Bessel function. Inserting these into (12) and changing the order of integration, we obtain

$$
\begin{aligned}
Y_{\text {decay }} & \equiv \int_{T_{0}}^{T_{R}} \frac{\left\langle\Gamma_{(I \rightarrow \chi+\cdots)}\right\rangle n_{I}}{s(T) H(T) T} d T \\
& =\frac{\bar{g} M_{P} \Gamma}{16 \pi^{2} m^{2}} \int_{t_{R}}^{\infty} d t \frac{1}{e^{t} \mp 1}\left[\left\{\frac{\pi}{2}-\tan ^{-1}\left(\frac{t_{R}}{\sqrt{t^{2}-t_{R}^{2}}}\right)\right\} t^{4}+t_{R}\left(t^{2}-2 t_{R}^{2}\right) \sqrt{t^{2}-t_{R}^{2}}\right], \\
Y_{\text {scat }} & \equiv \int_{T_{0}}^{T_{R}} \frac{\left\langle\sigma_{(I+J \rightarrow \chi+\cdots)} v\right\rangle n_{I} n_{J}}{s(T) H(T) T} d T \\
& =\frac{\bar{g} M_{P}}{16 \pi^{4}} \int_{t_{R}}^{\infty} d t t^{3} K_{1}(t) \int_{\left(m_{1}+m_{2}\right)}^{t T_{R}} d(\sqrt{s}) \sigma(s)\left[\frac{\left(s-m_{1}^{2}-m_{2}^{2}\right)^{2}-4 m_{1}^{2} m_{2}^{2}}{s^{2}}\right],
\end{aligned}
$$

where $\bar{g}=135 \sqrt{10} /\left(2 \pi^{3} g_{*}^{3 / 2}\right)$ and $t_{R}=m / T_{R}$ in $Y_{\text {decay }}$ and $\left(m_{1}+m_{2}\right) / T_{R}$ in $Y_{\text {Scat }}$. For $Y_{\text {scat }}$, the Maxwell-Boltzmann distribution is used for the simplicity of calculation, which gives the correct result up to a factor of order 1 .

For $T_{R}>m, Y_{\text {decay }}$ is well approximated by

$$
Y_{\text {decay }} \simeq \frac{3 \zeta(5) \bar{g} M_{P} \Gamma}{4 \pi m^{2}}
$$

For $Y_{\text {scat }}$, we present two typical examples. First, when $m_{1}, m_{2} \ll T_{R}$ and $\sigma(s)=\sigma_{0}=$ constant, $Y_{\text {scat }}$ is given by

$$
Y_{\mathrm{scat}}=\frac{\bar{g} \sigma_{0} M_{P} T_{R}}{\pi^{4}}
$$


For the second example with $m_{1}=M_{X}, m_{2}=0$ and $\sigma(s)$ of (17), we have

$$
\begin{aligned}
Y_{\text {scat }} & =\frac{\bar{g} \xi_{4} m_{\lambda}^{2} M_{X}}{3 \pi^{2} \alpha^{2} m_{3 / 2}^{2} M_{P}} \int_{t_{R}}^{\infty} d t K_{1}(t)\left[\frac{248}{45} t^{3}-8 t_{R} t^{2}+\frac{62}{9} t_{R}^{3}-\frac{2}{5} \frac{t_{R}^{5}}{t^{2}}-\left(t_{R} t^{2}-\frac{2}{3} t_{R}^{3}\right) \log \left(\frac{t^{4}}{t_{R}^{4}}\right)\right] \\
& \simeq \frac{124 \bar{g} \xi_{4} m_{\lambda}^{2} M_{X}}{45 \pi \alpha^{2} m_{3 / 2}^{2} M_{P}}, \quad \text { for } T_{R} \gg M_{X} .
\end{aligned}
$$




\section{REFERENCES}

[1] For a review, see H. P. Nilles, Phys. Rep. 110, 1 (1984).

[2] For a review, see G. F. Giudice and R. Rattazzi, hep-ph/9801271

[3] P. Fayet, Phys. Lett. B175, 471 (1986).

[4] T. Moroi, H. Murayama and M. Yamaguchi, Phys. Lett. B303, 289 (1993).

[5] A. de Gouviêa, T. Moroi and H. Murayama, Phys. Rev. D56, 1281 (1997).

[6] T. Lee, hep-ph/9812451.

[7] M. Dine and A. E. Nelson, Phys. Rev. D48, 1277 (1993); M. Dine, A. E. Nelson and Y. Shirman, Phys. Rev. D51, 1362 (1995); M. Dine, A. E. Nelson, Y. Nir and Y. Shirman, Phys. Rev. D53, 2658 (1996).

[8] K. Choi and K. Y. Lee, Phys. Rev. D54, 6591 (1996); E. Poppitz and S. P. Trivedi, Phys. Rev. D55, 5508 (1997); N. Arkani-Hamed, J. March-Russell and H. Murayama, Nucl. Phys. B509, 3 (1998).

[9] K. I. Izawa and T. Yanagida, Prog. Theor. Phys. 95, 829 (1996); K. Intriligator and S. Thomas, Nucl. Phys. B473, 121 (1996)

[10] T. Lee and G.-H. Wu, Phys. Lett. B447, 83 (1999).

[11] A. Brignole, F. Feruglio and F. Zwirner, JHEP 9711, 001 (1997).

[12] H. Pagels and J. R. Primack, Phys. Rev. Lett. 48, 223 (1982)

[13] J. Ellis, J. E. Kim and D. V. Nanopoulos, Phys. Lett. B145, 181 (1984); J. Ellis, G. B. Gelmini, J. L. Lopez, D. V. Nanopoulos and S. Sarkar, Nucl. Phys. B373, 399 (1992). 


\section{FIGURES}

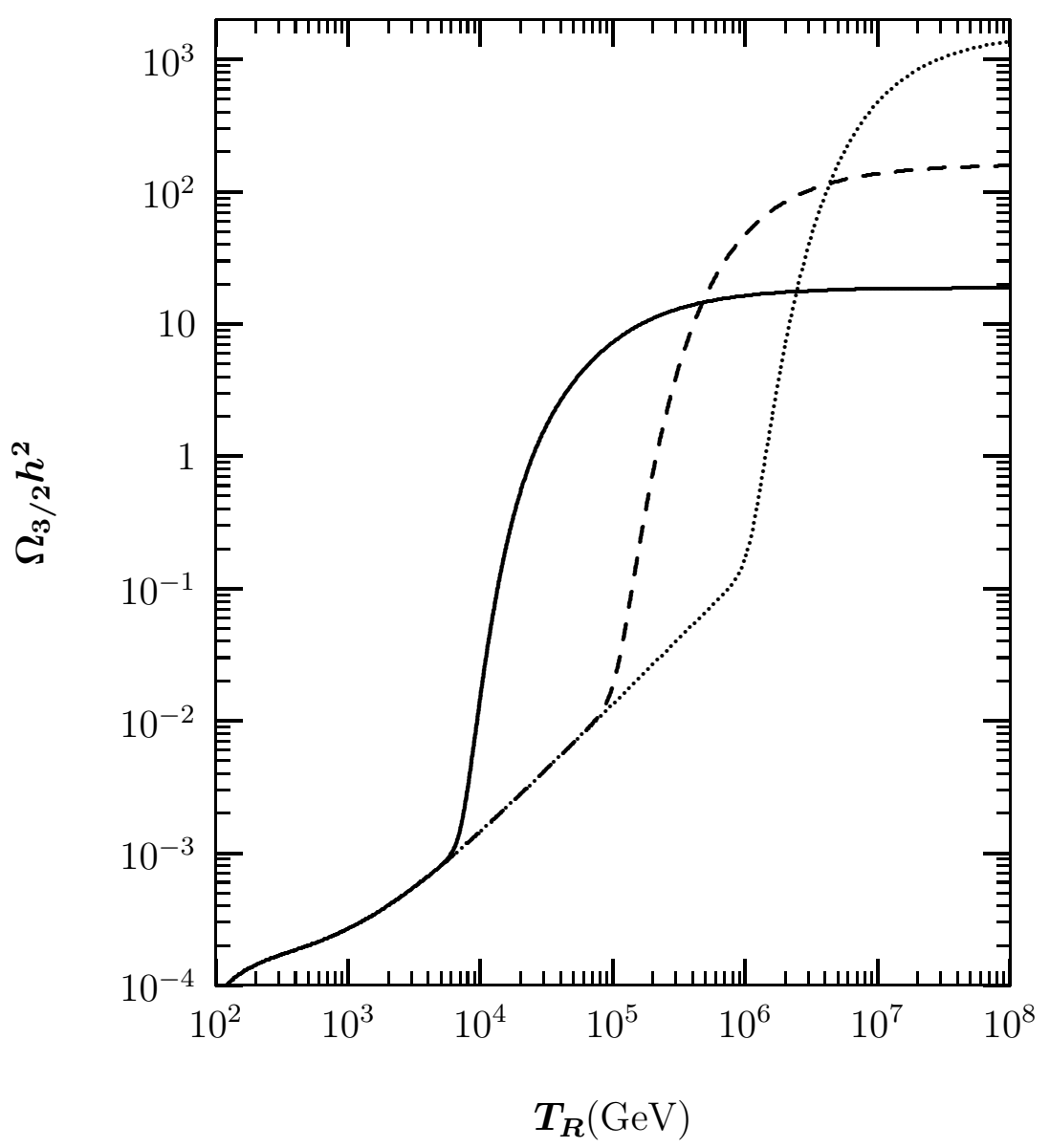

FIG. 1. $\Omega_{3 / 2} h^{2}$ as a function of the reheat temperature $T_{R}$ for the gravitino mass $m_{3 / 2}=10$ $\mathrm{MeV}$, the MSSM gaugino mass $m_{\lambda}=300 \mathrm{GeV}$ and the messenger mass $M_{X}=10^{5} \mathrm{GeV}$ (solid line), $10^{6} \mathrm{GeV}$ (dashed line), $10^{7} \mathrm{GeV}$ (dotted line). $\Omega_{3 / 2} h^{2}$ is scaled by $\left(10 \mathrm{MeV} / m_{3 / 2}\right)$ for different values of $m_{3 / 2}$. 

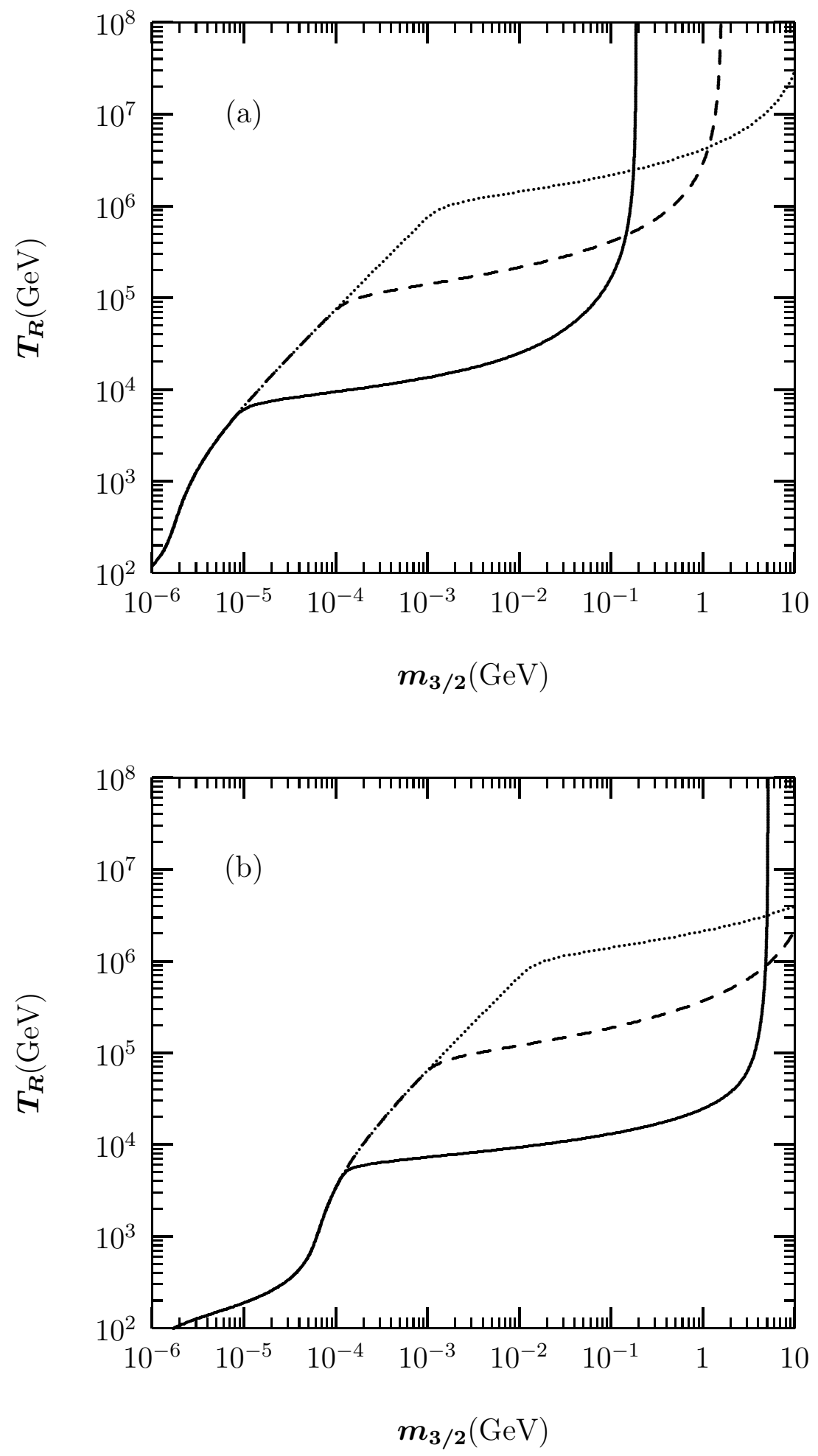

FIG. 2. The upper bound on the reheat temperature $T_{R}$ from $\Omega_{3 / 2} h^{2}<1$ as a function of the gravitino mass $m_{3 / 2}$ in the gauge mediated models. We used the MSSM gaugino mass $m_{\lambda}=300$ $\mathrm{GeV}$ in (a) and $m_{\lambda}=1 \mathrm{TeV}$ in (b), and the messenger mass $M_{X}=10^{5} \mathrm{GeV}$ (solid line), $M_{X}=10^{6}$ $\mathrm{GeV}$ (dashed line), $M_{X}=10^{7} \mathrm{GeV}$ (dotted line), respectively. 